\title{
Chronic kidney disease duration influences the renal graft outcome after living donor kidney transplantation. Myth or fact?
}

\section{Introduction}

- Chronic kidney disease (CKD) is a condition associated with high morbidity and mortality. Kidney transplantation is known to improve quality of life, and probably also longevity, with grafts from living donors providing the best prognosis.

- There is some concern about the waiting time before transplantation, due to the accumulation of cardiovascular comorbidities associated with chronic renal failure and dialysis.

- The aim of our study was to evaluate the influence of CKD duration on the outcome of renal function in living donor kidney transplanted patients (LDKT)

\section{Material and Methods}

- Retrospective observational audit of electronic medical records of LDKT from January 2010 to December 2012.

- Main outcome: glomerular filtration rate (GFR) at hospital discharge, and 1st, 3rd, 6th, 12th and 24th month postoperatively.

- Statistical analysis using IBM SPSS Statistics v.21.

- Spearman correlation was used to assess the relation between CKD duration and GFR (significance level $p<0.05$ ).

\section{Results}

\section{Demographic Variables}

Values presented as \%; Mean (maximum; minimum)

\begin{tabular}{|l|c|c|}
\hline & Donors & Recipients \\
\hline Male (\%) & $23,9 \%$ & $76,1 \%$ \\
\hline \hline Age (years) & $46(67 ; 20)$ & $39(70,15)$ \\
\hline
\end{tabular}

\section{Recipient Characterization}

Values presented as \%, or Median (maximum; minimum)

\begin{tabular}{|l|c|}
\hline ASA physical status III / IV (\%) & 21,$7 ; 78,3$ \\
\hline High Blood Pressure (\%) & 78,3 \\
\hline Dyslipidaemia (\%) & 34,8 \\
\hline Smoking (\%) & 28,3 \\
\hline Diabetes Mellitus (\%) & 8,7 \\
\hline Preemptive LDKT & $8.7 \%$ \\
\hline CKD duration (months) & $\mathbf{8 9 ( 3 1 2 ; 1 0 )}$ \\
\hline
\end{tabular}

CKD Duration : GFR

\begin{tabular}{|c|c|c|}
\hline Time & SC Coefficient & $p$-Value \\
\hline Discharge & -0.23 & 0.14 \\
\hline $1^{\text {st }}$ month & -0.15 & 0.32 \\
\hline $3^{\text {rd }}$ month & -0.18 & 0.23 \\
\hline $6^{\text {th }}$ month & -0.22 & 0.16 \\
\hline $12^{\text {th }}$ month & -0.25 & 0.10 \\
\hline $24^{\text {th }}$ month & -0.19 & 0.23 \\
\hline
\end{tabular}

\section{Conclusions}

- Duration of CKD was not associated with an impact on renal graft function from living donors, until at least two years postoperatively.

- Prospective and controlled studies are necessary to better clarify the impact of CKD duration on renal graft function received from living donors. 